\title{
Vibration of Tensegrity Stucture by Using SEM
}

\author{
Buntara Sthenly Gan ${ }^{1 *}$ and Shota Kiryu ${ }^{2}$ \\ ${ }^{1}$ Department of Architecture, College of Engineering, Nihon University, 1 Nakagawara, Tokusada, \\ Koriyama, Fukushima 963-8642, Japan \\ ${ }^{2}$ Department of Architecture, Graduate School of Engineering, Nihon University, 1 Nakagawara, \\ Tokusada, Koriyama, Fukushima 963-8642, Japan \\ * Corresponding author: buntara@ arch.ce.nihon-u.ac.jp
}

(Received: September 12 ${ }^{\text {th }}, 2019$; Accepted: October 26 $\left.6^{\text {th }}, 2019\right)$

\begin{abstract}
A tensegrity structure is a structure which consists both of compressive and tensile elements without being restrained at the boundaries. The self-equilibrium state inside the tensegrity structure is the condition that builds the structure without any boundary condition necessity. The conventional Eigensystem solver cannot deal with this kind of structure since there are rigid body motions in the governing equations. The exact dynamic solution of tensegrity structure problems can only be obtained by using the frequencydependent dynamic method. In this study, the free vibrational characteristics of a tensegrity structure which is modeled by a combination of the compressive strut and tensile cables elements are solved by using the Spectral Element Method (SEM). Natural frequencies of the tensegrity are tracked by using the WittrickWilliams algorithm. Numerical calculations are given to show the effectiveness, efficiency, and accuracy of the SEM in solving the axially vibrating members of the tensegrity structures.
\end{abstract}

Keywords: Frequency domain; Self-equilibrium; Spectral Element Method (SEM); Tensegrity Structure; Vibration

\section{Introduction}

Finite element method (FEM) is one of the major and common computational methods available in many fields of science and engineering. The vibration modes of a tensegrity vary depending on the vibration frequencies and its wavelengths. To obtain a sufficiently accurate dynamic response, all necessary high-frequency wave modes have to be considered in the analysis. The size of meshes must be sufficiently small to be used in the modeling. Because the conventional FEM is formulated based on the frequency-independent polynomial shape functions, thus the FEM cannot accommodate all essential high-frequency wave modes without fine element discretization. Hence, the FEM solution becomes less accurate, particularly at high frequencies, where the frequencies are related with short wavelengths.

The accuracy of the solution can be improved by using the shape functions, which depend on the natural frequency of the tensegrity. The shape functions that depend on the frequencies are known as the dynamic shape functions. The dynamic shape functions can be considered as necessity in high-frequency wave modes, hence it is not necessary to refine the element. This sophisticated concept has directed to the so-called dynamic stiffness method (DSM) [1,2]. Because the dynamic shape functions are formulated by using exact dynamic stiffness matrix, they treat the mass in a structure member implicitly. The DSM produces an infinite number of Eigensolutions by using the exact dynamic stiffness matrix represented by the least number of DOF. Alike with the formulation of the conventional finite element stiffness matrices, the stiffness of the exact 
dynamic matrices can be assembled similarly as in the FEM. Therefore, in the DSM, the procedures of elements meshing and assembling are very similar to the way in the FEM.

In spectral analysis method (SAM) [3], the solution to the governing differential equations can be achieved by adding an infinite number of wave modes with different frequencies. The scheme is very similar to finding the solutions of continuous Fourier transform. To obtain the time histories of the solutions, the inverse Fourier transform is applied to the infinite set of spectral components in the frequency domain. Only in the mathematically simple problems, the continuous Fourier transform is feasible. In practice, the discrete Fourier transform (DFT) is widely used for discontinuous Fourier transform.

Narayanan and Beskos combined the features of the SAM with those of the DSM by introducing the new concept of the spectral element method (SEM) [4]. As illustrated in Fig. 1, the SEM can be considered as the mixture of the main features of the FEM, SAM, and DSM.

The works in appliying the concept of SEM for analyzing the vibration problems of plate structures have been reported [5-7].

In this study, the free vibrations of tensegrities are analyzed by using SEM. The exact natural frequencies of the free vibration tensegrity are investigated.

\section{Kinematic of Axially Prestressed Cables}

Axially prestressed structures are of technologically important in a wide range of science and engineering problems. The typical examples of the axially prestressed structures can be found in the music instruments, in the textile industry, chain and belt drives, high-speed paper and magnetic tapes, aerial cable ropeways, cooling tower strips and many others. The axially prestressed of members of a structure like in a tensegrity can significantly affect its dynamic characteristics, giving effects to variations in natural frequencies and vibration modes. Thus it is important to accurately predict the dynamic characteristics of such axially prestressed structures to achieve safe design. If the bending stiffness of an axially prestressed is negligible, the structures can be represented by the string model. In this paper, spectral element modeling is considered for solving the axially prestressed members in a tensegrity.

\section{Dynamic Equilibrium Equation in Frequency Domain}

Fig. 2 shows a prestressed element under a constant force, which is positive for tension and negative for compression. The element is supported by two simple supports which are separated by a distance, and the element has the mass density and the uniform cross-sectional area.

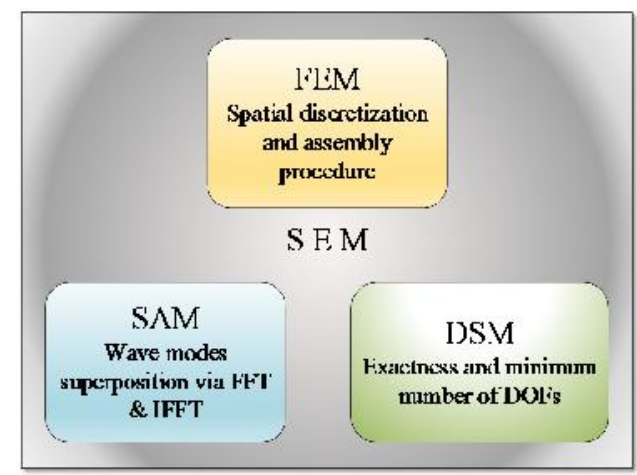

Fig. 1. Schematic diagram of the Spectral Element Method 


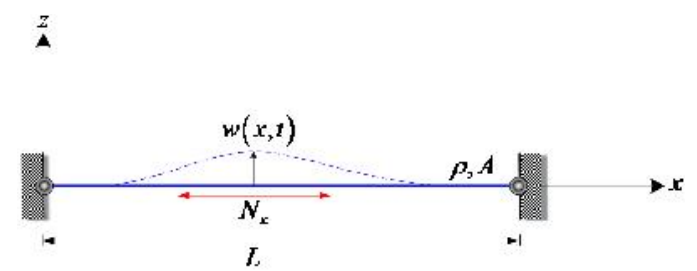

Fig. 2. Transversal displacement of an axially pre-stressed element.

The small transversal vibration and negligibly small flexural (bending) rigidity of the prestressed element are assumed. The extended form of Hamilton's principle for the systems of pre-stressed is used to derive the governing equation of motion and associated boundary conditions. The extended form of Hamilton's principle is given by

$$
\int_{t_{1}}^{t_{2}}(\delta K-\delta S+\delta V) d t=0
$$

where $K$ is the kinetic energy, $S$ is the strain energy, and $V$ is the virtual work done by nonconservative external forces. The equation of motion is then can be given by,

$$
\rho A \ddot{w}-N_{x} w^{\prime \prime}=f(x, t)
$$

\section{Spectral Element Formulation}

Assume the dynamic response of a structure in the spectral form as,

$$
w(x, t)=\frac{1}{N} \sum_{n=0}^{N-1} W_{n}(x) e^{i \omega_{n} t}
$$

where $W_{n}(x) e^{i \omega_{n} t}$ is the spectral components of the displacement $w(x, t), N$ is the sampling number and $\omega_{n}$ is the $n^{\text {th }}$ natural frequency.

The frequency-domain governing equation can be obtained by substituting the assumed displacement $w(x, t)$ into Eq. (2) which yields to,

$$
-\omega^{2} \rho A W-N_{x} W^{\prime \prime}=F(x)
$$

\section{Frequency Dependent Dynamic Shape Function}

By solving the homogeneous equation of Eq. (4), the frequency dependent dynamic shape function can be obtained as,

$$
\mathbf{N}(x ; \omega)=\mathbf{e}(x ; \omega) \mathbf{H}_{S}^{-1}(\omega)
$$

where,

$$
\begin{aligned}
\mathbf{H}_{S}(\omega) & =\left[\begin{array}{cc}
1 & 1 \\
e^{-i k_{1} L} & e^{-i k_{2} L}
\end{array}\right] \\
\mathbf{e}(x ; \omega) & =\left[\begin{array}{ll}
e^{-i k_{1} x} & e^{-i k_{2} x}
\end{array}\right]
\end{aligned}
$$

\section{Spectral Element Formulation}

By substituting the shape functions back into the governing equation in Eq. (4), the spectral element matrix can be obtained as follow.

$$
\mathbf{S}(\omega)=\left(\mathbf{H}_{S}^{-1}(\omega)\right)^{T} \mathbf{D}(\omega) \mathbf{H}_{S}^{-1}(\omega)
$$

where, 


$$
\mathbf{D}(\omega)=-N_{x} \mathbf{K}^{T} \mathbf{E}_{S} \mathbf{K}-\rho A \omega^{2} \mathbf{E}_{S}
$$

and,

$$
\begin{aligned}
& \mathbf{E}_{S}(\omega)=\int_{0}^{L}\left[\mathbf{e}^{T}(x ; \omega) \mathbf{e}(x ; \omega)\right] d x=E_{S R S} \\
& \overline{\mathbf{E}}_{S}(\omega)=\left.\mathbf{e}^{T}(x ; \omega) \mathbf{e}(x ; \omega)\right|_{0} ^{L}=\bar{E}_{S R S}
\end{aligned}
$$

with,

$$
E_{S R S}= \begin{cases}\frac{i}{k_{1}+k_{2}} \bar{E}_{S R S} & k_{1}+k_{2} \neq 0 \\ L & k_{1}+k_{2}=0\end{cases}
$$

at the boundaries becomes,

$$
\bar{E}_{S R S}=\mathbf{e}^{-i\left(k_{1}+k_{2}\right) L}-1
$$

\section{Free Vibration of a Triplex Tensegrity Structure}

Fig. 3 shows a prestressed triplex tensegrity example. The prestressed force is calculated from the multiplication of the tension coefficients obtained from the analysis of self-state with the scaling formula,

$$
\mathbf{N}_{x}=\mathbf{q} \times L \times 6000
$$

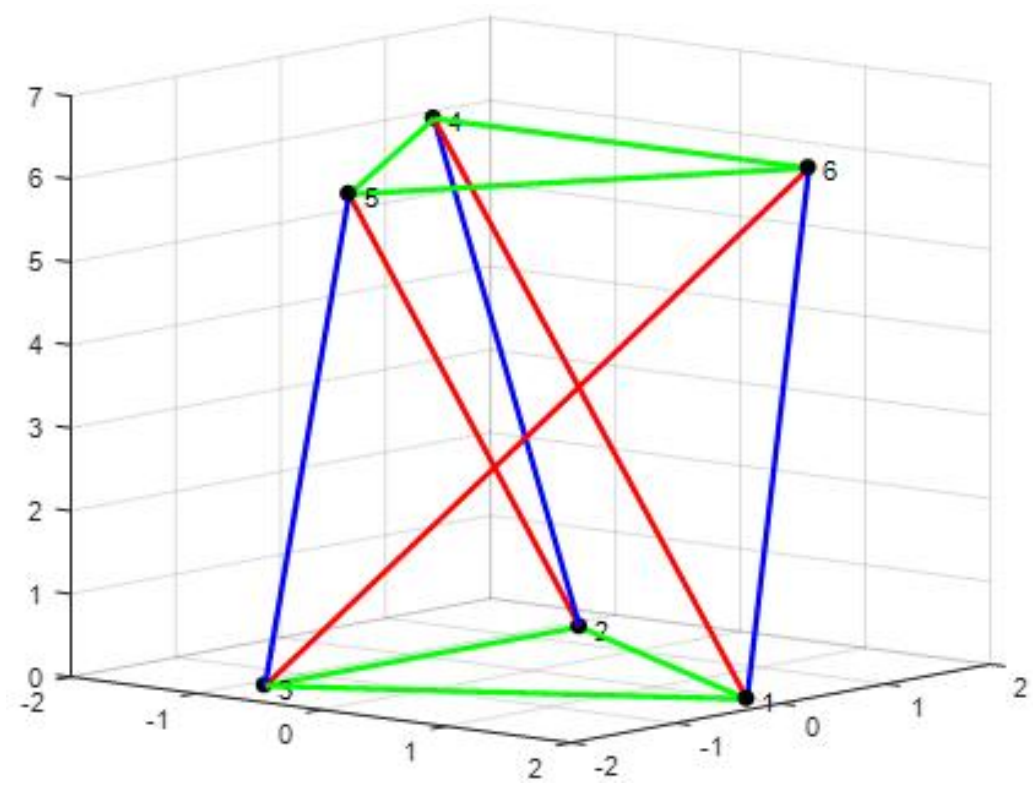

Fig. 3. Axially pre-stressed struts and cables of a Triplex Tensegrity Model. 


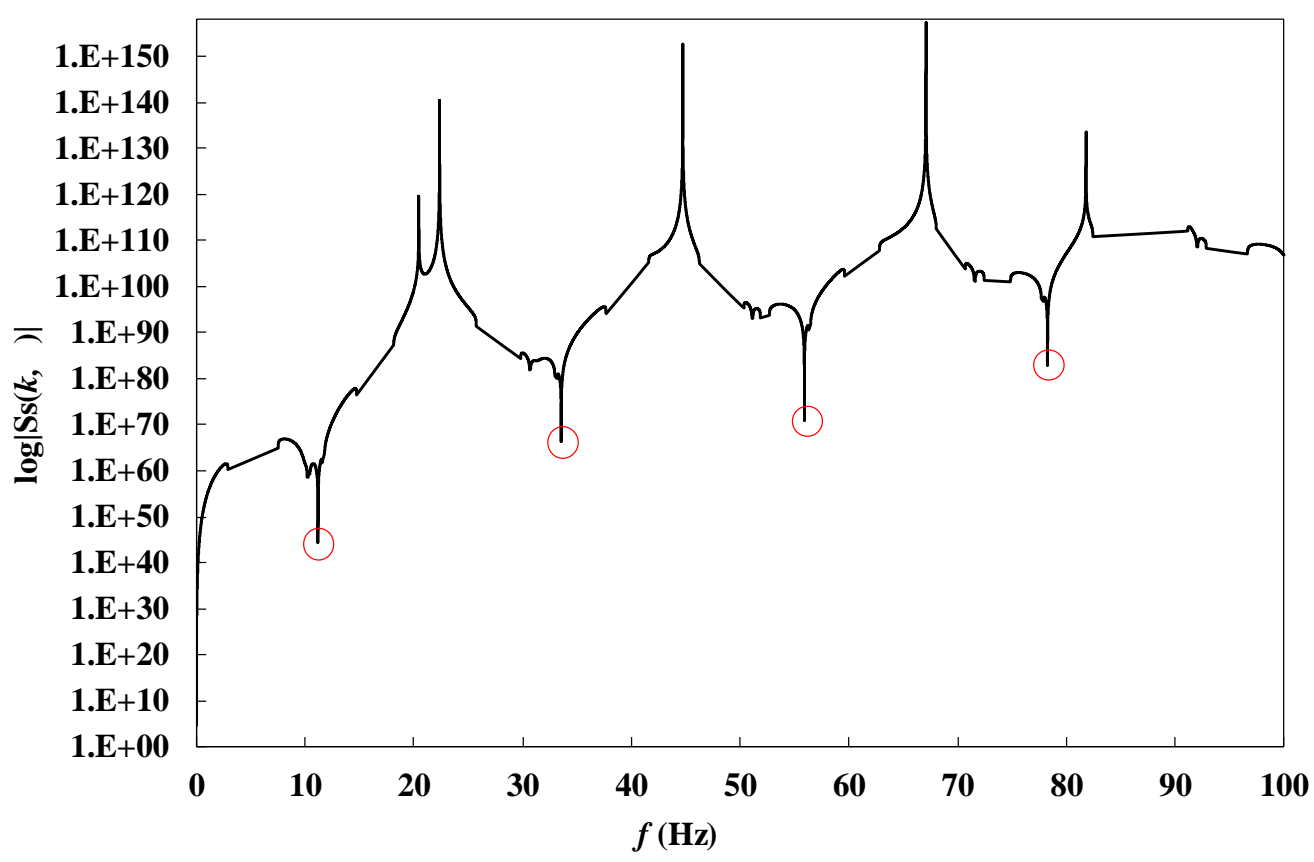

Fig. 4. The natural frequencies of axially pre-stressed triplex tensegrity example

After the coordinates transformation constructions and element assembling procedure, the results of the lowest four frequencies of the vibrating triplex tensegrity example are calculated. Table 1 and Fig. 4 depict the computed results of the first four lowest natural frequencies of the triplex tensegrity. The Wittrick-Williams algorithm [8] is implemented to calculate the natural frequencies of the triplex tensegrity.

Table 1. The first four natural frequencies of the vibrating triplex tensegrity

\begin{tabular}{ccccc}
\hline$(\mathrm{Hz})$ & $f_{1}$ & $f_{2}$ & $f_{3}$ & $f_{4}$ \\
\hline Triplex & 11.18 & 33.54 & 55.90 & 78.26 \\
\hline
\end{tabular}

It should be noted that in the computation the natural frequencies of the free vibrating problem of a Tensegrity, there is no need to have any nodal boundary constrained like in the FEM modeling. The singularities of the equilibrium matrix can be handled by using the SEM.

\section{Conclusions}

This paper has demonstrated that in order to obtain a sufficiently accurate dynamic response of unconstrained Tensegrity structure in space, the use of the dynamic shape functions which depend on the vibration frequencies and wavelengths are necessary.

In current study, the exact natural frequencies of the free vibration tensegrity can be computed even the rigid body of motion occurs in the structural system.

Numerical computations are shown to show the effectiveness, efficiency, and accuracy of the SEM.

\section{References}

[1] Banerjee, J.R. (1977). Dynamic Stiffness Formulation for Structural Elements: A General Approach. Computers \& Structures, 63(1), 101-103.

[2] Leung, A.Y.T. (1993). Dynamic Stiffness and Substructures, London: Springer-Verlag. 
[3] Doyle, J.F. (1997), Wave Propagation in Structures: Spectral Analysis Using Fast Discrete Fourier Transforms, New York: Springer.

[4] Narayanan, G.V. and Beskos, D.E. (1978). Use of Dynamic Influence Coefficients in Forced Vibration Problems with the aid of Fast Fourier transform. Computers \& Structures, 9(2), $145-150$.

[5] Kiryu, S. and Gan, B.S. (2018), Vibrational Analysis of Levy-Type Plates by Using SEM. Journal of Advanced Civil and Environmental Engineering, 1(1), 18-29.

[6] Kiryu, S., Alisjahbana, S.W., Alisjahbana, I., Nagao, M. and Gan, B.S. (2019), Free vibration of a Levy-type solution for plates based on two-variable refined plate theory by using SEM. Journal of Vibroengineering, 21(3), 726-735.

[7] Kiryu, S., Alisjahbana, S. W., Alisjahbana, I. and Gan, B. S., Free vibration of orthotropic levy-type solution plates by using SEM. The $7^{\text {th }}$ International Conference of Euro Asia Civil Engineering Forum, September 2019, University of Stuttgart, Germany.

[8] Wittrick, W.H. and Williams, F.W. (1971), A General Algorithm for Computing Natural Frequencies of Elastic Structures. Quarterly Journal of Mechanics and Applied Mathematics, 24(3), 263-284. 